\title{
Editorial: Recent Advances on Intelligent Mobility and Edge Computing
}

\author{
Xun Shao ${ }^{1} \cdot$ Zhi Liu $^{2} \cdot$ Xianfu Chen ${ }^{3} \cdot$ Seng W. Loke ${ }^{4} \cdot$ Hwee-Pink Tan ${ }^{5}$ \\ Published online: 22 March 2021 \\ (C) Springer Science+Business Media, LLC, part of Springer Nature 2021
}

In recent years, we have seen fast development of wireless communications, networking, and cloud computing: $4 \mathrm{G}, 5 \mathrm{G}$ and multiaccess networks greatly enhance the quality of service (QoS) of wireless access networks; software-defined networking, network function virtualization, and information-centric networking largely reduce the cost of network service providers and improve the quality of experience (QoE) of end-users; the development of mobile devices and mobile cloud computing lead to explosive deployment of mobile services and applications; the recent development of advanced algorithms such as Deep Learning has shown great potential in resource allocation and service orchestration. Deep integration of the above technologies provides a large number of opportunities for realizing the future mobile computing systems. On the other hand, to realize an efficient and sustainable ecosystem, many challenges exist. The challenges lie in a wide range, including devices technologies, communications and networking technologies, cloud and edge computing technologies, energy harvesting technologies, and incentive and marketing mechanisms. Among the abovementioned research directions, this special issue puts special focus on IoT and mobile edge computing (MEC), which is an essential component of the

Xun Shao

x-shao@ieee.org

1 School of Regional Innovation and Social Design Engineering, Kitami Institute of Technology, Kitami, Japan

2 University of Electro-Communications, Chofu, Japan

3 VTT Technical Research Centre of Finland Ltd, Oulu, Finland

4 School of Info Technology, Deakin University, Melbourne, Australia

5 Singapore Management University, 81 Victoria St, Singapore 188065, Singapore upcoming 5G architecture, and of fundamental importance to the future mobile computing systems.

This special issue features six selected papers with high quality. In the first article with the title "An Efficient Protocol for the Tag-information Sampling Problem in RFID Systems", the authors studied the tag-information sampling problem in RFID systems. They first obtained the theoretical lower bound of communication cost, and then designed an efficient protocol to approach the lower bound. It was proved that the proposed protocol achieves a communication cost within a factor of 2 of the theoretical lower bound.

In the second article with the title "An Optimal Uplink Scheduling in Heterogeneous PLC and LTE Communication for Delay-aware Smart Grid Applications", the authors first presented the analysis of the advantages and disadvantages of PLC and LTE communication, and then designed a network framework for PLC and LTE uplink communication in smart grid. Based on the network framework, the authors proposed an uplink scheduling transmission method for sampling data with optimized throughput according to the requirements of system delay and reliability.

In the next article with the title "Distributed Spectrum and Power Allocation for D2D-U Networks: A Scheme based on NN and Federated Learning", the authors studied the Device-to-Device communication on unlicensed bands (D2D-U) enabled network, and proposed a distributed joint power and spectrum allocation scheme in order to improve the spectrum efficiency (SE) on the unlicensed bands and fit its distributed structure while ensuring the fairness among D2D-U links and the harmonious coexistence with WiFi networks.

Smart health is widely regarded as one of the most promising technologies for the post-COVID-19 era. The fourth article titled "A Displacement Estimated Method for Real Time Tissue Ultrasound Elastography" proposed a novel displacement estimation method for real 
time tissue ultrasound elastography. The proposed method is composed with the quality-guided block matching module and the phase-zero search module, and proved to be efficient with extensive simulations.

With the recent development of the IoT technology and deep learning theory, human action recognition has been widely concerned and studied. The fifth article, "APFNet: Amplitude-Phase Fusion Network for CSI-based Action Recognition" proposed a novel lightweight action recognition model based on CSI amplitude-phase fusion. With the proposed method, the CSI of the WiFi signal is taken as the data, and the lightweight neural network APFNet is used to realize indoor human action recognition.

The last article titled "Neural Networks with Improved Extreme Learning Machine for Demand Prediction of Bikesharing" investigated the problems in accurate demand prediction of bike-sharing, and presented a novel prediction model based on the pseudo-double hidden layer feedforward neural networks. The performance is verified with extensive simulations with real-world dataset.

Acknowledgements The guest editors are thankful to our reviewers for their effort in reviewing the manuscripts. We also thank the Edit-in-Chief, Dr. Imrich Chlamtac for his supportive guidance during the entire process. The special issue is sponsored by Cooperative Research Project Program of the Research Institute of Electrical Communication, Tohoku University, the Telecommunications Advancement Foundation, Japan, and Kitami Institute of Technology, Japan.

Publisher's note Springer Nature remains neutral with regard to jurisdictional claims in published maps and institutional affiliations.

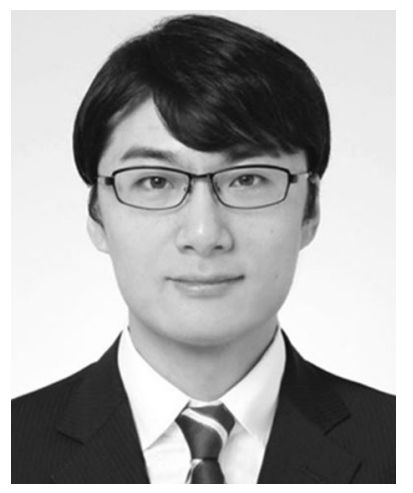

Xun Shao received his Ph.D. in information science from the Graduate School of Information Science and Technology, Osaka University, Japan, in 2013. From 2013 to 2017, he was a researcher with the National Institute of I $\mathrm{n} \mathrm{format}$ i o $\mathrm{n}$ a $\mathrm{nd}$ Communications Technology (NICT) in Japan. Currently, he is an Assistant Professor at the School of Regional Innovation and Social Design Engineering, Kitami Institute of Technology, Japan. His research interests include distributed systems, networking, and green computing. He is a member of the IEEE, IEICE, and IPSJ.

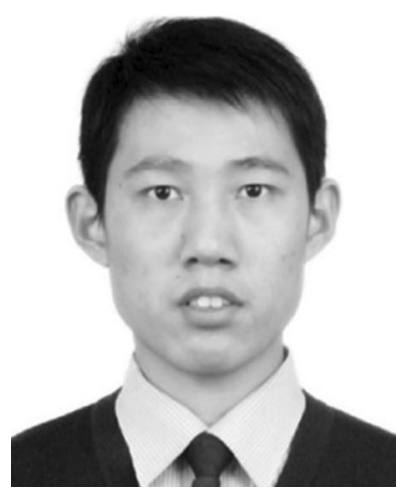

Zhi Liu received the B.E. degree from the University of Science and Technology of China, Hefei China and the Ph.D. degree in informatics from National Institute of Informatics, Tokyo, Japan. He is currently an Associate Professor with the University of Electro-Communications, Japan. His research interests include video network transmission, vehicular networks, and mobile edge computing. He is currently an editorial board member of Springer Wireless Networks and has been a Guest Editor of ACM/Springer Mobile Networks \& Applications, Springer Wireless Networks and IEICE Transactions on Information and Systems. He is a senior member of IEEE.

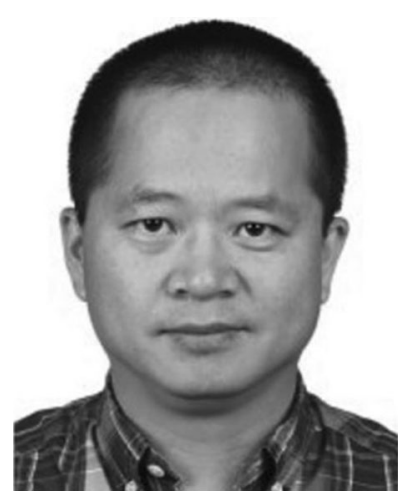

Xianfu Chen received the Ph.D. degree in signal and information processing from Zhejiang University, Hangzhou, China, in March 2012. Since April 2012, he has been with the VTT Technical Research Centre of Finland Ltd., Oulu, Finland, where he is currently a Senior Scientist. His research interests include various aspects of wireless communications and networking, with emphasis on human-level and artificial intelligence for resource awareness in next-

generation communication networks.

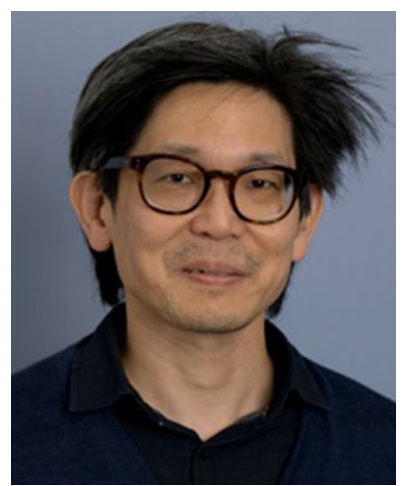

Seng W. Loke completed his $\mathrm{PhD}$ in 1998 at the University of Melbourne, and the BSc (1st class Hons) in 1994 at the Australian National University, winning a University Medal in Computer Science. He was an Honorary Associate of the Centre for Distributed Systems and Software Engineering, Monash University for several years (2006-2011). Currently, he is Professor in Computer Science at the School of Information Technology in Deakin University. He was Reader and Associate Professor at the Department of Computer Science and Information Technology in La Trobe University. He led the Pervasive Computing Lab at La Trobe. His research has mainly been in pervasive (ubiquitous) computing, mobile computing, and Internet of Things (IoT). Seng Loke is in the editorial board of the International Journal of Science and Engineering of Smart Vehicles, Springer Human-Centric Computing and Information Sciences, International Journal of Mobile Computing and Multimedia Communications, and the Sensors journal in the Internet-of-Things section. 


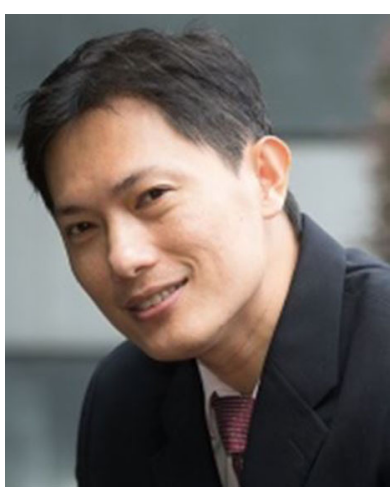

Hwee-Pink TAN graduated from the Technion, Israel Institute of Technology, Israel in August 2004 with a Ph.D. In December 2004, he was awarded the A *STAR International Fellowship to conduct postdoctoral research at Eindhoven University of Technology (Dec 2004 to Jun 2006) and Trinity College Dublin (Jul 2006 to Mar 2008) on the design and evaluation of wireless networks. He is currently an associate professor with Singapore Management University, Singapore. He is a Senior Member of the IEEE, has published more than 100 papers, and has served on executive roles for various conferences on IoT. 